\title{
La Perspectiva de Segunda Persona de la Atribución de Estados Mentales: Una Revisión Sistemática de su Estado Actual de Desarrollo
}

\section{The Second-Person Perspective of Mental States Attribution: A Systematic Review of its Current State of Development}

\author{
Maximiliano Vietri \\ Universidad Nacional de La Plata \\ Nicolás Alessandroni \\ Universidad Autónoma de Madrid \\ María Cristina Piro \\ Universidad Nacional de La Plata
}

\begin{abstract}
En psicología y filosofía de la mente el problema del conocimiento de las otras mentes es un campo de ricas disputas. Frente a las perspectivas de primera y tercera persona de la atribución mental que suponen que las mentes son opacas y que el acceso a las mismas es inferencial, la perspectiva de segunda persona postula la existencia de un modo no representacional de conocer las otras mentes, que emergería al interior de intercambios comunicativos tempranos bajo la forma de involucramientos emocionales naturales, básicos y directos. En este artículo de revisión sistemática se seleccionaron 57 manuscritos de la filosofía o la psicología con el objetivo de analizarlos y compararlos. Este trabajo permite justipreciar: (a) el estado actual de la perspectiva de segunda persona, (b) sus principales hallazgos en psicología y psicopatología del desarrollo, (c) las discusiones metodológicas que se derivan de su asunción y (d) un conjunto de críticas y cuestionamientos internos y externos formulados hacia ella. Se concluye que la perspectiva de segunda persona posee valor heurístico y se vería notablemente enriquecida si encarara la consideración cuidadosa de las críticas que la interpelan.
\end{abstract}

Palabras clave: perspectiva de segunda persona, atribución de estados mentales, cognición social, intersubjetividad, desarrollo cognitivo

\begin{abstract}
In psychology and philosophy of mind, the problem of knowledge of other minds is a field of rich disputes. Running counter to the perspectives of first- and third-person mental attribution, which assume that minds are opaque and that access to them is inferential, the second-person perspective postulates the existence of a non-representational way of knowing other minds, which is presumed to emerge within early communicative exchanges in the form of natural, basic, and direct emotional involvement. In this systematic review article, 57 philosophy or psychology manuscripts were selected with the aim of analyzing and comparing them. This study makes it possible to assess: (a) the current status of the second person perspective, (b) its main findings in developmental psychology and psychopathology, (c) the methodological discussions that stem from its assumption, and (d) a set of internal and external criticisms and questions formulated with respect to it. It is concluded that the second person perspective has a heuristic value and would be remarkably enriched if it carefully addressed the criticisms leveled against it.
\end{abstract}

Keywords: second-person perspective, mental states attribution, social cognition, intersubjectivity, cognitive development

Explicar la atribución de estados mentales (AEM) reviste gran importancia para la psicología y la filosofía. Primero, porque nuestra capacidad para comprender las conductas propias y ajenas depende de concebir que los demás y nosotros mismos tenemos estados mentales (Melot \& Nadel, 1998/2003). Esto implica que podamos describir, explicar y predecir las acciones de nuestros congéneres vía la AEM y que podamos adaptar nuestra propia acción a los contextos interactivos. Segundo, por la intersección que existe entre la AEM y la psicopatología. Existen casos clínicos en los que se evidencian dificultades vinculadas con la atribución

Maximiliano Vietri y María Cristina Piro, Laboratorio de Investigaciones en Psicoanálisis y Psicopatología, Facultad de Psicología, Universidad Nacional de La Plata, Argentina; Nicolás Alessandroni, Departamento Interfacultativo de Psicología Evolutiva y de la Educación, Facultad de Psicología, Universidad Autónoma de Madrid, España.

La correspondencia relativa a este artículo debe ser dirigida a Maximiliano Vietri, Laboratorio de Investigaciones en Psicoanálisis y Psicopatología, Universidad Nacional de La Plata, Calle 51 e/122 y 123 s/n, Ensenada (CP 1925), Buenos Aires, Argentina. E-mail: mvietri@psico.unlp.edu.ar 
mentalista. Tal es el caso de los trastornos del espectro autista (American Psychiatric Association, 2013; World Health Organization, 2010). Comprender cómo llegamos a conocer la mente de los demás posee relevancia para tratar de entender las mentes de aquellos sujetos con trayectorias atípicas de desarrollo (Karmiloff-Smith, 1994) y, eventualmente, plantear posibilidades de intervención psicológica.

En psicología y filosofía de la mente la atribución mentalista fue tradicionalmente abordada por dos perspectivas: la perspectiva de tercera persona y la perspectiva de primera persona. La perspectiva de tercera persona plantea que los sujetos típicos contarían con una teoría de la mente (Gopnik \& Wellman, 1994/2002; Leslie, 1987, 2000; Premack \& Woodruff, 1978). La teoría de la mente consistiría en un conjunto de generalizaciones de tipo legaliforme que se expresa utilizando conceptos psicológicos acerca de la cognición y la motivación que es aplicable a otros y a nosotros mismos. Dicho repertorio conceptual sería puesto en funcionamiento para explicar, predecir y describir las conductas propias y ajenas. A partir de dicho conocimiento general, se podrían generar razonamientos deductivos acerca de lo que otro individuo cree o desea para poder alcanzar conclusiones sobre las acciones que realizó o que realizará (Balmaceda, 2014). La perspectiva de primera persona propone que la cognición social está regida por mecanismos de simulación (Goldman, 1989; Goldman \& Mason, 2007; Gordon, 1986; Heal, 1986). Representar la conducta de otra persona - y los procesos mentales involucrados en ella- se lograría produciendo conductas y procesos similares en nuestras mentes, pero sin que lleguen a efectivizarse. Es decir, si nos ponemos en el lugar del otro y determinamos cuáles son sus estados mentales en ese momento, podríamos entender qué es lo que está haciendo o podríamos predecir lo que hará (Balmaceda, 2014).

Sin embargo, estas teorías fueron objetivo de distintas críticas. Una de las principales críticas que se ha esgrimido en contra de ellas es que para explicar la atribución mentalista ambas parten considerando a los sujetos como individuos aislados, solipsistas. Esta concepción, denominada "paradigma del aislamiento" (Schilbach et al., 2013, p. 394), lleva a considerar la cognición social en términos de un observador desapegado que observa y lee los estados mentales de otra persona, la que, a su vez, no se ve afectada por este hecho y no puede reaccionar ante dicha lectura. En otras palabras:

(...) El problema radica en el compromiso de la psicología con la brecha epistémica, la cual, creemos, deriva del hecho de concebir al sujeto cognoscente como fundamentalmente aislado, lo que requiere una mediación - una idea, una teoría, una revelación, un concepto- para superar la brecha entre el sí mismo y el otro o (...) la brecha entre el comportamiento y la mente. (Reddy \& Morris, 2004, p. 648).

Esto quiere decir que las mentes (incluso la propia) nos resultarían opacas a la hora de intentar conocerlas y que para lograrlo necesitaríamos de un "puente" que permita sortear la distancia entre ellas. Al concebir las mentes como entidades internas, invisibles y no materiales, que gobiernan e influyen sobre la conducta y que solo pueden ser inferidas, estas teorías reeditan el dualismo cartesiano mente/cuerpo (Costall, Leudar \& Reddy, 2006; Leudar \& Costall, 2004; Reddy, 2001a).

Una segunda crítica proviene del hecho de que ambas postulan la imposibilidad ontogenética de acceder al terreno de las atribuciones mentalistas hasta haber desarrollado una capacidad representacional. Hasta dicho momento (2-4 años, según Baron-Cohen, 1985), los niños serían incapaces de conocer otras mentes (Baron-Cohen, 1985). Todo esto conduce a una discontinuidad aguda en el desarrollo de la lectura intencional. Así, en un primer momento los niños solo percibirían comportamientos sin sentido y luego, al contar con recursos representacionales, se tornarían capaces de inferir mentes detrás de los comportamientos. Sin embargo, existe evidencia empírica que contradice esta postura discontinuista y plantea la necesidad de analizar qué ocurre en el terreno de la atribución mentalista antes de la adquisición ontogenética de habilidades representacionales (véase la sección Lo que Nos Enseñan los Psicólogos).

Un tercer cuestionamiento a las perspectivas ortodoxas proviene de los planteos de Schilbach et al. (2013), quienes sostienen que las teorías de primera y de tercera persona son subsidiarias de una concepción espectatorial de la cognición social. Las visiones espectatoriales se han desarrollado dentro del paradigma de la ciencia cognitiva ortodoxa (Burdman, 2015; Shapiro, 2011), que entiende la cognición como procesamiento pasivo de información provista por el mundo. Al interior de este paradigma, la acción de los sujetos en el mundo tiene una importancia secundaria (no constitutiva) y el rol del cuerpo se limita a la función de conducción de estímulos del entorno hacia la mente, considerada esta última como una dimensión abstracta y amodal (Piccinini, 2007).

Considerando las criticas antes mencionadas es que, en las últimas décadas, algunos psicólogos y filósofos han propuesto un punto de vista alternativo que podría dar respuesta a los interrogantes que las perspectivas ortodoxas dejan sin resolver: la perspectiva de segunda persona (P2P). En ese contexto, revisar las 
explicaciones sobre el origen y desarrollo de la atribución mentalista a la luz de los aportes de la P2P es altamente relevante, primero, por el impacto conceptual que esto podría conllevar a nivel del desarrollo típico de la atribución mentalista y, segundo, por el impacto conceptual y práctico que esto podría tener a nivel de las trayectorias de desarrollo atípico (véase supra).

En atención a lo antedicho, nos propusimos efectuar una revisión sistemática de un conjunto de aportaciones teóricas y empíricas vinculadas con la P2P. En esta revisión privilegiamos el análisis de literatura psicológica, aunque también desarrollamos brevemente algunos aportes asociados a la filosofía de la mente, dada la importancia que estos últimos importan para el estudio de la cognición social.

\section{Método}

Nuestro trabajo constituye una revisión sistemática de literatura académica. Para su elaboración hemos adoptado como marco metodológico las propuestas de la declaración PRISMA para la realización y publicación de revisiones sistemáticas y metaanálisis (Urrútia \& Bonfill, 2010). El objetivo de nuestro estudio fue analizar y comparar un conjunto de estudios teóricos y empíricos vinculados a la P2P de la AEM en función de los resultados y las premisas epistemológicas y teóricas que subyacen a ellos.

\section{Procedimiento}

El curso de nuestra toma de decisiones durante la recolección y análisis de los datos fue el siguiente:

Recopilación de datos. Realizamos una búsqueda bibliográfica, consultando las bases de datos más importantes del área [ISI (Web of Science), Scopus, Proquest, ERIH, PubMed, ScienceDirect, PsycINFO, Psicodoc, Dialnet, SciELO, Latindex Catálogo y NBR], a través de las siguientes palabras clave: perspectiva de segunda persona (second person perspective), teoría del involucramiento (engagement theory), teoría de la interacción (interaction theory), intersubjetividad (intersubjectivity) y cognición social (social cognition). En la búsqueda consideramos las palabras clave de manera individual y combinada. La búsqueda arrojó un total de entre 209 y 28.904 publicaciones, dependiendo del idioma de la búsqueda y la base de datos que consultamos en cada caso. Debido a que dichas cantidades de publicaciones no resultan manejables en el contexto de una revisión sistemática y que no todos los resultados constituían aportes relevantes, filtramos las publicaciones, de acuerdo a un conjunto de criterios que señalamos a continuación:

Selección de estudios. Realizamos un proceso de selección siguiendo los siguientes criterios de elegibilidad:

1. Tipo de publicación: publicaciones teóricas o empíricas, incluyendo artículos, capítulos de libros y libros. No tuvimos en cuenta tesis, tesinas, ponencias o conferencias.

2. Objeto de estudio: características de los procesos de involucramiento en el contexto de interacciones diádicas (sujeto-sujeto) y su vinculación con la AEM. Por ende, prescindimos de otras publicaciones que abordan la temática considerando otros objetos de estudio.

3. Enfoque teórico/epistemológico: publicaciones que se abocan al estudio del objeto definido anteriormente, adoptando la P2P, tal como esta es definida por la mayoría de las publicaciones del área (véase Alessandroni, Vietri \& Krasutzky, 2017; Alessandroni, Vietri \& Piro, 2016). No consideramos los estudios que mencionaban las palabras clave o el objeto de estudio, pero que se encontraban filiados a otros marcos teóricos/epistemológicos, por ejemplo, Gallese (2014).

4. Área de publicación: filosofía de la mente, psicología y psicopatología. No consideramos publicaciones de subáreas de la psicología o la filosofía irrelevantes para este estudio (i.e., psicología laboral o filosofía de la ciencia) ni específicas de otras áreas (i.e., neurociencias, lingüística o educación).

5. Idioma: consideramos únicamente publicaciones cuyo cuerpo central estuviese escrito en inglés o español.

6. Fecha de publicación: manuscritos publicados entre 2000 y 2017. No obstante, también incluimos referencias a escritos anteriores que constituyen antecedentes sustanciales para la temática de la revisión (véanse, por ejemplo, Hobson, Ouston \& Lee, 1988; Schutz, 1962).

7. Duplicados: excluimos los manuscritos duplicados.

Se podría argumentar que los trabajos provenientes de la neurociencia social podrían formar parte de este trabajo. Tal es el caso de trabajos como los de Mojzisch et al. (2006), Schilbach, Eickhoff, Cieslik, Kuzmanovic y Vogeley (2012), Schilbach et al. (2006), Schilbach et al. (2010) y Schilbach et al. (2011). Sin embargo, 
hemos decidido no incluirlos en este estudio por dos razones. En primer lugar, por el hecho de que un análisis que haga justicia a la complejidad teórica y metodológica de estos experimentos excedería los límites de nuestro estudio (centrado en psicología). En segundo lugar, por el hecho de que estas disciplinas difieren tanto en sus objetos de estudio como en sus métodos de abordaje. Por lo tanto, esta discrepancia teórico/metodológica tornaría forzada su comparación.

\section{Análisis de Datos}

Luego de la lectura, identificamos dimensiones de análisis relevantes para la tarea comparativa y la elaboración de la discusión. Circunscribimos las siguientes dimensiones: características principales del involucramiento (engagement) en las interacciones diádicas, relación del involucramiento con la AEM y características de la atribución mentalista. Para las publicaciones empíricas consideramos dimensiones adicionales: subárea disciplinar, unidades de análisis, procedimiento, tipo de diseño, objeto de estudio, variables, características de la muestra, contexto de la investigación y conclusiones más relevantes. Presentamos algunas de estas dimensiones de manera sintética en las Tablas 1 y 2.

\section{Resultados}

Nuestra selección arrojó un total de 57 referencias: seis de ellas fueron publicadas antes de 2000, 26 en el período 2000-2009 y 25 en el período 2010-2017.

\section{Lo que Nos Enseñan los Filósofos}

Algunos filósofos contemporáneos de la mente han concebido a la P2P como una propuesta atractiva para superar las perspectivas de primera y tercera persona (Gomila, 2001; Hutto, 2008) y para explicar los avatares de la atribución mentalista en algunos casos de actividades humanas que no se ajustan completamente a las perspectivas ortodoxas (Pérez, 2013). Gomila (2008), por ejemplo, plantea que existe una forma no reflexiva ni teórica de acceso a las mentes que no requeriría inferencias, conceptos ni capacidades representacionales y que estaría basada en el reconocimiento emocional y recíproco de configuraciones corporales expresivas e intencionales (expresiones faciales y corporales, disposiciones posturales, gestos y otros patrones conductuales) que funcionan como claves o señales.

Se trataría de una modalidad que operaría de forma básica, directa e inmediata (Gallagher, 2001) en contextos públicos y sociales (Pérez \& Español, 2014) y, más específicamente, en el seno de los tempranos intercambios diádicos cara a cara (Gomila, 2002). Este vínculo entre la P2P y el desarrollo temprano ha llevado a algunos filósofos a afirmar que la primera podría caracterizarse como una forma primitiva de comunicación intencional (Scotto, 2002).

El tipo de conocimiento que nos brindaría esta modalidad sería, además, irreductible a aquel que se obtendría a través de AEM de primera y tercera persona (Yeo, 2016), característica asociada a dos factores: (a) en este tipo de atribución mentalista no existen un observador de mentes y una mente pasiva que es examinada por el observador, sino dos participantes activos enlazados por la consideración de la relación práctica que los convoca y (b) este tipo de atribución mentalista se desarrolla como una forma de habilidad (Balmaceda, 2014) y no como una forma de razonamiento proposicional (i.e., como un modo de know-how y no de know-that; Klin, Jones, Schultz \& Volkmar, 2003). De esta forma, la P2P, en tanto perspectiva orientada por la acción (Dominey et al., 2016), quita a los estados mentales de la esfera de la mente entendida como dimensión oculta o abstracta y los traslada a la esfera pública de la acción interactiva intersubjetiva y recíproca.

En la P2P el foco está puesto en aquellas interacciones en las cuales se da un involucramiento emocional y corporal. Para algunos filósofos, la AEM de segunda persona es un caso de intercorporeidad (Gallagher, 2016).

Resulta relevante señalar doce aspectos que, de acuerdo con nuestro análisis, condensan los fundamentos de los argumentos presentes en los estudios desglosados supra y en otros estudios importantes (Balmaceda, 2014; Español, 2007; Ferrer de Luna, 2014; Gallagher, 2004, 2008; Gomila, 2001, 2002, 2008; Pedace, 2012; Pérez, 2013; Pérez \& Español, 2014; Pérez \& Lawler, 2017, Scotto, 2002):

1. Es directa y pre-teórica: las configuraciones expresivas son percibidas como significativas sin necesidad de interpretación; no requieren de la mediación de ninguna teoría ni mecanismo de simulación.

2. Es básica e inmediata: involucra procesos cognitivos fenomenológicamente impenetrables que se apoyan en procesos subpersonales de nivel básico. 
3. No supone una actividad mental "meta": no hay un estado mental sobre del estado mental del otro, sino un estado mental originado por el estado mental del otro y así sucesivamente.

4. Es implícita: el resultado de la atribución mentalista no se formula conscientemente.

5. Es recíproca: los participantes de una interacción realizan atribuciones simultáneas considerando la evolución de la dinámica interactiva.

6. Es perceptible: los participantes se dan cuenta de las atribuciones mutuas que tienen lugar en contextos interactivos.

7. Es reactiva y espontánea: surge como respuesta emocional inevitable ante las conductas del otro en contextos interactivos.

8. Es práctica: el sentido de las atribuciones es mediar la interacción.

9. Es dinámica: la comprensión mutua se desarrolla como un proceso, es decir, como desenvolvimiento longitudinal.

10. No tiene pretensiones explicativas o predictivas: se orienta a dar sentido a la conducta propia y ajena.

11. Es ontogenéticamente primaria: precede a (y propiciaría el surgimiento de) las modalidades de primera y de tercera persona de AEM.

12. No es excluyente: podrían existir vínculos entre esta modalidad de AEM y las modalidades descriptas por las perspectivas de primera y tercera persona.

\section{Lo que Nos Enseñan los Psicólogos}

Teorizaciones sobre la AEM en desarrollo típico. En psicología del desarrollo existen importantes antecedentes dedicados a tratar el surgimiento ontogenético de la intersubjetividad y las características del conocimiento de las otras mentes en la temprana infancia (véanse, entre otros, Benjamin, 1990; Cohn \& Tronick, 1987; Hubley \& Trevarthen, 1979; Kaye \& Fogel, 1980; Mahler, Pine \& Bergman, 1975/1977; Ranly, 1966; Schutz, 1962; Spitz, 1965/1998; Stern, 1985/2005; Trevarthen, 1979, 1984; Zahn-Waxler \& RadkeYarrow, 1990). Sin embargo, a partir de la difusión de la propuesta de la P2P, la indagación de estos tópicos se ha visto enriquecida.

Así, por ejemplo, Hobson $(1990,2002)$ defiende que el involucramiento social signado por un nexo emocional entre los sujetos es clave para el origen del pensamiento, tanto a nivel ontogenético como filogenético. Según él, es la comunicación afectiva con los demás la que permite a los bebés tener las primeras formas de experiencia de las otras mentes (véase también Reddy, 2001a, 2003, 2010). Desde la perspectiva de Reddy, el involucramiento genuino con los demás no solo es una vía de acceso privilegiada al conocimiento de los demás, sino que además "crea mentes" (Reddy \& Morris, 2004, p. 660). En sus palabras:

Lo 'público' —el compartir — es un paso necesario en el desarrollo para permitir lo 'privado' —lo oculto-, no al revés. Incluso podría ser que el involucramiento genuino —el contacto psicológico mutuo - pueda guiarnos a una comprensión del self que no hubiera sido posible sin él. (Reddy, 2010, p. 16).

$\mathrm{El}$ involucramiento que propone la $\mathrm{P} 2 \mathrm{P}$ es una forma de acceso a la mentalidad basada en componentes emocionales que prescinde de la capacidad representacional y de toda forma de inferencia (Leudar \& Costall, 2004), puesto que dicho conocimiento es públicamente accesible, es decir, directamente perceptible como significativo en la conducta del otro (Reddy \& Uithol, 2016). De este modo, el dualismo cartesiano que distingue tajantemente res cogitans de res extensa es interpelado. Aquí, los componentes conductuales asociados a la emoción (por ejemplo, gestos faciales o disposiciones posturales) no son considerados distintos de los componentes mentales: "la mente se vuelve el modo [énfasis en el original] en que un cuerpo viviente actúa, no algo separable de, escondido detrás de o guiando a sus acciones" (Reddy, 2010, p. 14).

En línea con este enfoque, Zahavi y Parnas (2003) plantean que las experiencias no son internas, sino que están presentes en los gestos y acciones corporales. Por ello, en las relaciones cara a cara puede tener lugar una comprensión directa y pragmática de los elementos expresivos de la conducta del otro. Desde su punto de vista, no percibimos primero un cuerpo físico para luego inferir la existencia de subjetividad; la comprensión intersubjetiva implica el encuentro con un todo unificado: psique-cuerpo. Desde este paradigma, las emociones no son parte constitutiva del mundo interior de individuos aislados, sino que son patrimonio de agentes activa, conmovedora y asertivamente vinculados con el mundo y con los otros: las emociones son estados compartidos, cuya naturaleza y función es suscitar respuestas simpáticas en otros (Reddy, 2001a). El 
carácter público y reactivo de las emociones, junto con la obligatoriedad de la respuesta que estas evocan, constituye uno de los fundamentos para la producción de interacciones recíprocas (Reddy \& Trevarthen, 2004). Esto revela el carácter dialógico del compromiso emocional, en el que cada participante regula los actos de los otros y viceversa.

Por último, vale la pena notar que en otros artículos (Buhrmann \& Di Paolo, 2017; De Jaegher \& Di Paolo, 2007; De Jaegher, Di Paolo \& Gallagher, 2010; Di Paolo \& De Jaegher, 2015; Fantasia, De Jaegher \& Fasulo, 2014) es posible encontrar una intersección entre las teorías corporeizadas de la mente (embodiment) y la P2P. Por ejemplo, De Jaegher y Di Paolo (2007) sostienen que el conocimiento de la mente de los demás no reside en mecanismos o capacidades inferenciales individuales, sino que depende (y tiene lugar al interior) de ciertos procesos interactivos y enactivos en los que los individuos participan creando sentidos (participatory sense-making). De esta manera, abandonan la esfera individual como ámbito explicativo de la cognición social para situarse en un terreno de negociación intersubjetiva de significados, lo que constituye una P2P. Así, la cognición social parecería involucrar un tipo particular de know-how interactivo, que nos permite interactuar con los demás, formar relaciones, comprendernos y actuar juntos (De Jaegher et al., 2010).

En este marco conceptual, el constructo interacción social halla su contrapartida operacional en el concepto de coordinación, concebida aquella como la correlación mecánica emergente que se establece entre los comportamientos de dos o más sistemas que están en acoplamiento y que puede ser explicada en términos de coordinación de fases dinámicas (Thelen \& Smith, 1996). De esta manera, se postula que las interacciones cognitivas pueden ser entendidas como procesos físicos y pueden ser descriptas recurriendo a ciertos modelos matemáticos. Así, en el involucramiento social los agentes coordinarían mecánicamente sus actividades de creación de sentido, tomando partido en las actividades de creación de sentido del otro. En otras palabras, la comprensión social se entiende como la generación-transformación de sentido en la interacción.

Hemos señalado hasta aquí una serie de elementos: (a) el carácter expresivo e intencional de la conducta, (b) la naturaleza compartida y conmovedora de la emoción, (c) la obligatoriedad de respuesta ante la emoción del otro y (d) la reciprocidad de las atribuciones y el condicionamiento que ella ejerce sobre las atribuciones mismas. Estos rasgos determinan un giro epistemológico respecto de la unidad de análisis tradicionalmente considerada en psicología para estudiar la cognición social. La P2P cuestiona los límites del sujeto, sosteniendo que el estudio de las interacciones sociales debe considerar una unidad de análisis diferente a la sumatoria de dos individuos aislados. Se trata de estudiar, por el contrario, el devenir de la interacción misma de la que ellos forman parte en su conjunto. Como afirman Schilbach et al. (2013), una parte del conocimiento sobre el otro reside en la dinámica interactiva entre los agentes. Sugieren, de esta forma, que no existe un abismo epistémico absoluto entre el yo y el otro como sugieren las perspectivas clásicas, sino que la dinámica de la interacción social contribuye, y a veces constituye, nuestra consciencia de otras mentes. Este aspecto ya había sido destacado por Reddy (2010) cuando afirmó que:

La línea entre experimentarse uno mismo y percibir al otro es, al menos, permeable. Dentro del involucramiento emocional activo, la percepción de los otros siempre involucra la experiencia propioceptiva de los sentimientos-propios-por-los-otros y la propiocepción siempre involucra la percepción de los sentimientos-de-los-otros-por-uno-mismo. (p. 30).

Estudios empíricos en desarrollo típico. En psicología del desarrollo se han llevado adelante estudios empíricos desde la P2P con niños con desarrollo típico (ver Tabla 1).

Estos estudios proponen, en líneas generales, que en contextos de interacción intersubjetiva tiene lugar una serie de conductas que demuestran la existencia de una comprensión recíproca entre el niño y el adulto, de tipo intencional, enactiva y no inferencial, aportando, así, evidencia favorable de la P2P. Por ejemplo, Bordoni et al. (2016) plantearon como objetivo generar situaciones de interacción que permitieran comparar los efectos que la imitación y el entonamiento afectivo de adultos tienen en la reacción social del bebé, durante la segunda mitad del primer año de vida. La novedad metodológica más importante de este estudio longitudinal, cuasi-experimental y ecológico (hogar) es que se eligió que fuera la investigadora quien interactuara con los bebés. Desde este nuevo punto de vista, la atribución mentalista es analizada atendiendo a procesos interactivos de involucramiento intersubjetivo, en los que el mismo investigador es quien interactúa con el sujeto investigado. Esto permite un equilibrio entre lo imprevisible de toda interacción y el control necesario en una investigación. En este estudio se concluye que el entonamiento afectivo produce menos miradas que otras modalidades interactivas (Bordoni et al., 2016). 
Tabla 1

Estudios Empíricos en Desarrollo Típico

\begin{tabular}{|c|c|c|c|c|}
\hline Referencia & Tipo de diseño & Objeto de estudio & Muestra & Conclusiones más relevantes \\
\hline $\begin{array}{l}\text { Ambrosini et al. } \\
\text { (2013) }\end{array}$ & $\begin{array}{l}\text { Descriptivo, } \\
\text { intensivo }^{1} \text {, } \\
\text { multivariado, } \\
\text { transeccional }^{2} \text {. }\end{array}$ & $\begin{array}{l}\text { Relación entre la } \\
\text { anticipación de los } \\
\text { objetivos de la acción } \\
\text { observada y la producción } \\
\text { de acciones. }\end{array}$ & $\begin{array}{l}3 \text { grupos de } 11 \text { niños } \\
\text { cada uno, de } 6,8 \text { y } 10 \\
\text { meses de edad. }\end{array}$ & $\begin{array}{l}\text { Desde los } 6 \text { meses los niños } \\
\text { anticipan con la mirada los } \\
\text { objetivos de los gestos de agarrar } \\
\text { (reaching). Esta habilidad se } \\
\text { vincula con su capacidad para } \\
\text { producir dichos gestos. }\end{array}$ \\
\hline $\begin{array}{l}\text { Bordoni, } \\
\text { Español \& De } \\
\text { Grande (2016) }\end{array}$ & $\begin{array}{l}\text { Descriptivo, } \\
\text { intensivo, } \\
\text { multivariado, } \\
\text { longitudinal. }\end{array}$ & $\begin{array}{l}\text { Efecto del entonamiento } \\
\text { afectivo y la imitación en } \\
\text { el involucramiento visual } \\
\text { infantil. }\end{array}$ & $\begin{array}{l}16 \text { bebés ( } 7 / 9) \text { observados } \\
\text { a sus } 6,9 \text { y } 12 \text { meses de } \\
\text { edad. }\end{array}$ & $\begin{array}{l}\text { El entonamiento afectivo provoca } \\
\text { los valores más bajos de } \\
\text { involucramiento visual en el bebé. }\end{array}$ \\
\hline $\begin{array}{l}\text { Fantasia, } \\
\text { Markova, } \\
\text { Fasulo, Costall } \\
\text { \& Reddy (2015) }\end{array}$ & $\begin{array}{l}\text { Descriptivo, } \\
\text { intensivo, } \\
\text { multivariado, } \\
\text { transversal. }\end{array}$ & $\begin{array}{l}\text { Sensibilidad a los } \\
\text { cambios en la secuencia } \\
\text { de alzamiento. }\end{array}$ & $\begin{array}{l}23 \text { bebés (10/13) de } 3 \\
\text { meses de edad. }\end{array}$ & $\begin{array}{l}\text { Los bebés de } 3 \text { meses son sensibles } \\
\text { a los cambios en la secuencia de } \\
\text { alzamiento. }\end{array}$ \\
\hline $\begin{array}{l}\text { Fantasia, } \\
\text { Fasulo, Costall } \\
\text { \& López (2014) }\end{array}$ & $\begin{array}{l}\text { Descriptivo, } \\
\text { intensivo, } \\
\text { multivariado, } \\
\text { transversal. }\end{array}$ & $\begin{array}{l}\text { Sensibilidad a los } \\
\text { cambios en los juegos. }\end{array}$ & $\begin{array}{l}20 \text { bebés (10/10) de } 3 \\
\text { meses de edad. }\end{array}$ & $\begin{array}{l}\text { Los bebés de } 3 \text { meses son sensibles } \\
\text { a los cambios en las condiciones del } \\
\text { juego. }\end{array}$ \\
\hline $\begin{array}{l}\text { Newton, Reddy } \\
\& \text { Bull (2000) } \\
\text { [Estudio 1] }\end{array}$ & $\begin{array}{l}\text { Descriptivo, de } \\
\text { caso único, } \\
\text { multivariado, } \\
\text { longitudinal. }\end{array}$ & $\begin{array}{l}\text { Comportamiento } \\
\text { engañoso cotidiano. }\end{array}$ & $\begin{array}{l}24 \text { niños de entre } 3 \text { y } 4 \\
\text { años de edad. }\end{array}$ & $\begin{array}{l}\text { Los sujetos emplearon varios tipos } \\
\text { de engaños, a pesar de no superar } \\
\text { la tarea de falsa creencia. }\end{array}$ \\
\hline $\begin{array}{l}\text { Newton et al., } \\
(2000) \text { [Estudio } \\
2]\end{array}$ & $\begin{array}{l}\text { Descriptivo, de } \\
\text { caso único, } \\
\text { multivariado, } \\
\text { longitudinal. }\end{array}$ & $\begin{array}{l}\text { Comportamiento } \\
\text { engañoso cotidiano. }\end{array}$ & $\begin{array}{l}1 \text { niño de } 2 \text { años } 5 \\
\text { meses de edad } \\
\text { observado durante } 25 \\
\text { semanas. }\end{array}$ & $\begin{array}{l}\text { El niño usa gran variedad de } \\
\text { conductas de engaño en } \\
\text { situaciones interactivas. }\end{array}$ \\
\hline Reddy (2000) & $\begin{array}{l}\text { Descriptivo, } \\
\text { intensivo, } \\
\text { multivariado, } \\
\text { longitudinal. }\end{array}$ & $\begin{array}{l}\text { Expresiones de la } \\
\text { timidez en la temprana } \\
\text { infancia. }\end{array}$ & $\begin{array}{l}5 \text { bebés de } 7 \text { semanas de } \\
\text { edad, observados } \\
\text { semanalmente hasta } \\
\text { sus } 20 \text { semanas de } \\
\text { edad. }\end{array}$ & $\begin{array}{l}\text { Los bebés sonríen tímidamente } \\
\text { desde los } 2 \text { meses de edad. Estas } \\
\text { sonrisas surgen en contextos } \\
\text { interactivos de atención social. }\end{array}$ \\
\hline $\begin{array}{l}\text { Reddy (2001b) } \\
\text { [Estudio 1] }\end{array}$ & $\begin{array}{l}\text { Descriptivo, } \\
\text { intensivo, } \\
\text { multivariado, } \\
\text { longitudinal. }\end{array}$ & $\begin{array}{l}\text { Creación de } \\
\text { involucramientos } \\
\text { humorísticos. }\end{array}$ & $\begin{array}{l}12 \text { niños visitados } \\
\text { quincenalmente desde } \\
\text { los } 7 \text { a los } 12 \text { meses de } \\
\text { edad. }\end{array}$ & $\begin{array}{l}\text { Desde los } 8 \text { meses los niños crean } \\
\text { involucramientos humorísticos. }\end{array}$ \\
\hline $\begin{array}{l}\text { Reddy (2001b) } \\
\text { [Estudio 2] }\end{array}$ & $\begin{array}{l}\text { Descriptivo, } \\
\text { intensivo, } \\
\text { multivariado, } \\
\text { longitudinal. }\end{array}$ & $\begin{array}{l}\text { Creación de } \\
\text { involucramientos } \\
\text { humorísticos. }\end{array}$ & $\begin{array}{l}20 \text { niños visitados a sus } \\
8 \text { y } 11 \text { meses de edad. }\end{array}$ & $\begin{array}{l}\text { Desde los } 8 \text { meses los niños crean } \\
\text { involucramientos humorísticos. }\end{array}$ \\
\hline
\end{tabular}


Tabla 1 (conclusión)

Estudios Empíricos en Desarrollo Típico

\begin{tabular}{|c|c|c|c|c|}
\hline Referencia & Tipo de diseño & Objeto de estudio & Muestra & Conclusiones más relevantes \\
\hline $\begin{array}{l}\text { Reddy, Liebal, } \\
\text { Hicks, } \\
\text { Jonnalagadda \& } \\
\text { Chintalapuri }\end{array}$ & $\begin{array}{l}\text { Descriptivo, } \\
\text { intensivo, } \\
\text { multivariado, } \\
\text { longitudinal. }\end{array}$ & $\begin{array}{l}\text { Relación entre directivas } \\
\text { parentales y el } \\
\text { surgimiento de la } \\
\text { obediencia en niños } \\
\text { pequeños. }\end{array}$ & $\begin{array}{l}\text { Interacciones de } 9 \\
\text { familias británicas y } 13 \\
\text { familias indias, filmadas } \\
\text { cuando los niños tenían } \\
6,5,8,0,9,5,11,0 \text { y } 12,5 \\
\text { meses de edad. }\end{array}$ & $\begin{array}{l}\text { El surgimiento de la obediencia } \\
\text { depende de procesos interactivos } \\
\text { facilitados por directivas adultas } \\
\text { redundantes, vinculadas a acciones } \\
\text { específicas. }\end{array}$ \\
\hline $\begin{array}{l}\text { Reddy, Markova } \\
\text { \& Wallot (2013) } \\
\text { [Estudio 1] }\end{array}$ & $\begin{array}{l}\text { Descriptivo, } \\
\text { intensivo, } \\
\text { multivariado, } \\
\text { transversal. }\end{array}$ & $\begin{array}{l}\text { Ajustes corporales } \\
\text { anticipatorios. }\end{array}$ & $\begin{array}{l}18 \text { bebés de } 3 \text { meses de } \\
\text { edad. }\end{array}$ & $\begin{array}{l}\text { La mayoría de los bebés exhibe } \\
\text { cambios posturales globales y } \\
\text { sistemáticos cuando su madre se } \\
\text { acerca para alzarlos. }\end{array}$ \\
\hline $\begin{array}{l}\text { Reddy, Markova } \\
\text { et al. (2013) } \\
\text { [Estudio 2] }\end{array}$ & $\begin{array}{l}\text { Descriptivo, } \\
\text { intensivo, } \\
\text { multivariado, } \\
\text { longitudinal. }\end{array}$ & $\begin{array}{l}\text { Ajustes corporales } \\
\text { anticipatorios. }\end{array}$ & $\begin{array}{l}10 \text { bebés de } 2 \text { meses de } \\
\text { edad, observados hasta } \\
\text { sus } 4 \text { meses de edad. }\end{array}$ & $\begin{array}{l}\text { Los ajustes posturales globales y } \\
\text { sistemáticos están presentes desde } \\
\text { los } 2 \text { meses de edad y son más } \\
\text { diferenciados a los } 4 \text { meses de edad. }\end{array}$ \\
\hline $\begin{array}{l}\text { Somerville, } \\
\text { Woodward \& } \\
\text { Needham (2005) }\end{array}$ & $\begin{array}{l}\text { Descriptivo, } \\
\text { extensivo, } \\
\text { multivariado, } \\
\text { transversal. }\end{array}$ & $\begin{array}{l}\text { Sensibilidad de los bebés } \\
\text { a las acciones basadas en } \\
\text { objetivos. }\end{array}$ & $\begin{array}{l}30 \text { bebés de } 3 \text { meses de } \\
\text { edad. }\end{array}$ & $\begin{array}{l}\text { Los bebés pueden detectar acciones } \\
\text { basadas en objetivos y emplear ese } \\
\text { conocimiento en la percepción de } \\
\text { acciones ajenas. }\end{array}$ \\
\hline \multicolumn{5}{|c|}{$\begin{array}{l}\text { Notas. }{ }^{1} \text { De acuerdo con Ynoub (2015), los diseños de investigación pueden clasificarse en intensivos o extensivos de acuerdo al tamaño de su muestra } \\
\text { Según esta autora, los estudios intensivos poseen una muestra pequeña, que no supera los } 20 \text { o } 30 \text { casos, mientras que los estudios extensivos poseen una } \\
\text { muestra grande o poblacional. Con base en ello, optamos por llamar "intensivos" a los estudios que, sin ser de caso único, hayan tenido muestras menores } \\
\text { a } 30 \text { casos. Los diseños cuyas muestras fuesen superiores a } 30 \text { casos los denominamos "extensivos". } \\
{ }^{2} \text { La diferencia entre los estudios transversales y transeccionales radica en la cantidad de grupos muestrales o cohortes. En los transversales, los sujetos } \\
\text { son semejantes en todas las variables de interés y conforman un grupo muestral uniforme. En los estudios transeccionales, los sujetos son asignados a una } \\
\text { de varias cohortes en función de sus diferencias en una variable clave (usualmente la edad). Así, en un estudio transeccional se podrían crear tres cohortes } \\
\text { de sujetos por edad (e.g., cohortes de } 6,8 \text { y } 10 \text { meses de edad, respectivamente). Debe notarse que la separación en cohortes no privilegia, a priori, ningún } \\
\text { tratamiento de la temporalidad. Por ejemplo, a cada cohorte se la podría observar en un único momento o en varios (e.g., una vez al mes durante } 6 \text { meses) } \\
\text { (Van der Maas, \& Molenaar, 1996) }\end{array}$} \\
\hline
\end{tabular}

En el estudio de Somerville et al. (2005), por ejemplo, se concluye que los niños de 3 meses comprenden acciones basadas en objetivos, sin contar con una capacidad representacional, y en otros estudios se aboga por la existencia de una comprensión intencional por parte de los niños (Newton et al, 2000; Reddy, 2000; Reddy, Markova et al., 2013). En otro de los estudios relevados, Reddy (2000) indagó las expresiones de la timidez en la temprana infancia. La metodología empleada involucró el registro multimedial de interacciones adulto-bebé en contextos ecológicos (hogar). La autora concluye que los bebés de 2 meses evidencian formas de timidez similares (funcional y estructuralmente) respecto de las manifestaciones de timidez de los adultos. Un dato significativo es que, a diferencia de lo ocurrido con los adultos, en los que la timidez es provocada por contextos evaluativos, en niños las sonrisas tímidas surgieron solo cuando ellos eran objetos de atención de los adultos. Esto indica que desde muy temprano los niños pueden reconocerse como objetos de la atención de los otros, pero solo en contextos interactivos. La hipótesis que avanza Reddy es que este resultado se debe a que el involucramiento permite el acceso a un tipo de información social que de otro modo no sería asequible. Pero, más importante aún, indica que el compromiso emocional propiciaría emociones autoconscientes que, posteriormente, podrían dar lugar a la fundación misma del pensamiento y del yo del sujeto.

En otro de los estudios, Newton et al. (2000) indagaron, a través del análisis interaccional, la habilidad para engañar de niños preescolares. En el primer diseño, la evidencia fue recolectada por las mismas madres de los niños, previamente instruidas con el fin de maximizar la confiabilidad y validez de los registros. Además, se realizaron entrevistas semiestructuradas con las madres después de cada observación, intentando obtener mayor información sobre los incidentes registrados por ellas con anterioridad. Durante cada una de las tres fases de observación, a cada niño se le administró una batería de tareas de falsa creencia. Esta tarea constituye el paradigma de investigación empírica empleado por quienes adhieren a la perspectiva de tercera persona. A pesar de no haber exhibido buen rendimiento en la tarea de falsa creencia, todos los 
sujetos emplearon varios tipos de engaño. A lo largo del estudio fueron registrados 14 tipos distintos de engaño, como, por ejemplo, "negación de maldad", "ocultamientos" y "falsas afirmaciones de desconocimiento". Algunos de estos engaños eran motivados (es decir, suscitados por una pregunta del adulto, por ejemplo) y otros espontáneos. En cuanto a los motivos de estos engaños, los autores indican que ellos son variados y abarcan desde la evasión al reproche hasta la simple diversión, y no se limitan a objetivos materiales o a situaciones apremiantes. En el segundo diseño del estudio, longitudinal de caso único, una de las investigadoras observó sistemáticamente a su propio hijo entre sus 24 y 30 meses de edad. A pesar de algunas ligeras diferencias, la metodología fue la misma que en el estudio anterior. Se registraron 37 episodios en los que el niño empleó algún tipo de engaño, a pesar de haber fallado tres de cuatro veces en la tarea de falsa creencia administrada.

Los resultados de estos diseños muestran que el engaño en niños pequeños es generalizado y razonablemente frecuente, responde a motivaciones variadas y ocurre tanto espontáneamente como en respuesta al comportamiento de los demás. Ello contradice abiertamente a las teorías ortodoxas de la cognición social que plantean que el desarrollo del engaño intencional sucede al descubrimiento de las creencias en torno a los 4 años de edad (véase, por ejemplo, Sodian, Taylor, Harris \& Perner, 1991; Stouthamer-Loeber, 1986; Wimmer \& Perner, 1983).

Aquí, los resultados son concluyentes: los niños pequeños emplean varias formas de engaño, a pesar de no haber superado la tarea de falsa creencia, y ello debe ser considerado un indicador de que ellos han comprendido la intencionalidad adulta. Dicho de otro modo, si un niño miente para evitar ser castigado, es porque ha comprendido que sería reprendido a partir del reconocimiento de elementos gestuales y no gestuales que se ponen en juego de forma enactiva en las situaciones de interacción y compromiso emocional (e.g., tono de voz fuerte de un adulto, ceño fruncido).

Todos los estudios sistematizados en la Tabla 1 sugieren que en el involucramiento tiene lugar una comprensión intencional de la conducta del otro que es anterior al desarrollo de la capacidad representacional en los niños. Esto implica que la cognición social se da desde muy temprano en la vida y tiene vínculos funcionales y estructurales con la percepción y la acción conjunta.

Estudios empíricos en desarrollo atípico: el caso del autismo. En la psicopatología del desarrollo también se emprendieron investigaciones empíricas desde la P2P, específicamente en autismo. Estos estudios se orientaron a desentrañar las particularidades de los intercambios sociales en los que se involucran los sujetos con autismo (ver Tabla 2). Para ello, consideraron variables tales como el grado de conexión con los demás, la fluidez interactiva o la calidad expresiva de las acciones realizadas por los sujetos con autismo.

En uno de los estudios, Reddy et al. (2002) indagan sobre las características del humor y la risa en niños preescolares. De acuerdo con los investigadores, estos son fenómenos relacionales centrales al interior de los intercambios sociales tempranos (tienen lugar a partir de los 3 meses de edad) que pueden dar cuenta de las habilidades de los niños para involucrarse con los demás y comprender sus estados intencionales. El diseño incluyó el registro multimedial y el análisis de escenas interactivas adulto/niño ocurridas en contextos ecológicos (hogar) y la realización de entrevistas semiestructuradas a los padres de cada niño. El análisis cuali-cuantitativo de los datos consideró las siguientes variables: episodios de risa (momento en el que cualquiera de los sujetos riera), duración de la risa (para el bebé o para el adulto), iniciativa de la risa, dirección de la risa y responsividad del niño a la risa de los demás. Además, se tuvieron en cuenta los contenidos graciosos de cada momento interactivo, y el tipo de contexto más amplio en el cual se enmarcó cada interacción. Los resultados indican que los niños con autismo y con síndrome de Down se ríen de cosas muy diferentes en las interacciones cotidianas. Los niños con autismo parecen no encontrar graciosos los actos sociales inapropiados ni las caras graciosas, mientras que estos sí son graciosos para los niños con síndrome de Down. Además, se observó que un 88\% de los niños con autismo suelen reírse de modo espontáneo y sin razón aparente. Los resultados de las entrevistas señalan que solo el 36\% de los niños con autismo suele intentar hacer reír a los demás, mientras que en los casos de sujetos con síndrome de Down esta misma conducta es exhibida por el $81 \%$ de los niños. 
Tabla 2

Estudios Empíricos en Desarrollo Atípico

\begin{tabular}{|c|c|c|c|c|}
\hline Referencia & Tipo de diseño & Objeto de estudio & Muestra & Conclusiones más relevantes \\
\hline $\begin{array}{l}\text { Dawson, Hill, } \\
\text { Spencer, } \\
\text { Galpert \& } \\
\text { Watson (1990) }\end{array}$ & $\begin{array}{l}\text { Descriptivo, } \\
\text { intensivo, } \\
\text { multivariado, } \\
\text { transversal. }\end{array}$ & $\begin{array}{l}\text { Uso de la mirada, } \\
\text { conductas sociales } \\
\text { y afectivas en el } \\
\text { autismo. }\end{array}$ & $\begin{array}{l}16 \text { sujetos con autismo (3/13) de } \\
\text { entre } 30 \text { y } 70 \text { meses de edad y } 16 \\
\text { sujetos con desarrollo típico (3/13). }\end{array}$ & $\begin{array}{l}\text { Los sujetos con autismo combinan } \\
\text { menos risas y miradas que los con } \\
\text { desarrollo típico. Sus madres sonríen } \\
\text { menos que estas. }\end{array}$ \\
\hline $\begin{array}{l}\text { García-Pérez, } \\
\text { Lee \& Hobson } \\
(2007)\end{array}$ & $\begin{array}{l}\text { Descriptivo, } \\
\text { intensivo, } \\
\text { multivariado, } \\
\text { transversal. }\end{array}$ & $\begin{array}{l}\text { Comunicación no } \\
\text { verbal e } \\
\text { involucramiento } \\
\text { afectivo. }\end{array}$ & $\begin{array}{l}12 \text { adolescentes con autismo de } 15 \\
\text { años de edad y } 12 \text { sujetos con } \\
\text { retraso mental de } 14 \text { años de } \\
\text { edad. }\end{array}$ & $\begin{array}{l}\text { El involucramiento afectivo fue menor } \\
\text { y menos fluido en sujetos con autismo } \\
\text { que en sujetos con retraso mental. }\end{array}$ \\
\hline $\begin{array}{l}\text { Hobson et al. } \\
\text { (1988) }\end{array}$ & $\begin{array}{l}\text { Descriptivo, } \\
\text { intensivo, } \\
\text { multivariado, } \\
\text { transversal. }\end{array}$ & $\begin{array}{l}\text { Reconocimiento } \\
\text { emocional en } \\
\text { caras } \\
\text { fotografiadas. }\end{array}$ & $\begin{array}{l}17 \text { adolescentes con autismo de } \\
\text { entre } 13 \text { y } 25 \text { años de edad y } 17 \\
\text { sujetos con retraso mental de } \\
\text { entre } 12 \text { y } 25 \text { años de edad. }\end{array}$ & $\begin{array}{l}\text { El reconocimiento emocional } \\
\text { disminuye más en sujetos con autismo } \\
\text { que en sujetos con retraso mental, } \\
\text { cuando se reducen las señales de } \\
\text { emoción en los rostros. }\end{array}$ \\
\hline $\begin{array}{l}\text { Hobson \& Lee } \\
(1998)\end{array}$ & $\begin{array}{l}\text { Descriptivo, } \\
\text { intensivo, } \\
\text { multivariado, } \\
\text { transversal. }\end{array}$ & $\begin{array}{l}\text { Saludos } \\
\text { solicitados y } \\
\text { espontáneos } \\
\text { dirigidos a un } \\
\text { adulto } \\
\text { desconocido. }\end{array}$ & $\begin{array}{l}24 \text { sujetos con autismo de entre } 8 \\
\text { y } 21 \text { años de edad y } 24 \text { sujetos con } \\
\text { retraso mental de entre } 8 \text { y } 23 \\
\text { años de edad. }\end{array}$ & $\begin{array}{l}\text { Los sujetos con autismo son menos } \\
\text { propensos que los sujetos con retraso } \\
\text { mental a emplear gestos verbales y no } \\
\text { verbales espontáneos de saludo y a } \\
\text { establecer contacto visual. }\end{array}$ \\
\hline $\begin{array}{l}\text { Hobson \& Lee } \\
\text { (1999) }\end{array}$ & $\begin{array}{l}\text { Descriptivo, } \\
\text { intensivo, } \\
\text { multivariado, } \\
\text { transversal. }\end{array}$ & $\begin{array}{l}\text { Habilidad para la } \\
\text { imitación diferida } \\
\text { del estilo de una } \\
\text { acción. }\end{array}$ & $\begin{array}{l}16 \text { sujetos con autismo de entre } 9 \\
\text { y } 18 \text { años de edad y } 16 \text { sujetos con } \\
\text { retraso mental no autistas de } \\
\text { entre } 11 \text { a } 17 \text { años de edad. }\end{array}$ & $\begin{array}{l}\text { Los sujetos con autismo imitan menos } \\
\text { el estilo de la acción y presentan gran } \\
\text { dificultad en acciones autodirigidas } \\
\text { que los con retraso mental. }\end{array}$ \\
\hline $\begin{array}{l}\text { Hobson, } \\
\text { Harris, } \\
\text { García- Pérez } \\
\text { \& Hobson } \\
(2009)\end{array}$ & $\begin{array}{l}\text { Descriptivo, } \\
\text { intensivo, } \\
\text { multivariado, } \\
\text { transversal. }\end{array}$ & $\begin{array}{l}\text { Desarrollo } \\
\text { temprano de la } \\
\text { empatía. }\end{array}$ & $\begin{array}{l}20 \text { sujetos con autismo de entre } 8 \\
\text { y } 15 \text { años de edad, } 18 \text { sujetos con } \\
\text { retraso mental de entre } 6 \text { y } 14 \\
\text { años de edad y } 14 \text { niños con } \\
\text { desarrollo típico de entre } 5 \text { y } 6 \\
\text { años de edad. }\end{array}$ & $\begin{array}{l}\text { Los sujetos con autismo muestran } \\
\text { menos signos de preocupación ante } \\
\text { una persona angustiada que los } \\
\text { sujetos con retraso mental y con } \\
\text { desarrollo típico. }\end{array}$ \\
\hline $\begin{array}{l}\text { Reddy, } \\
\text { Williams \& } \\
\text { Vaughan } \\
(2002)\end{array}$ & $\begin{array}{l}\text { Descriptivo, } \\
\text { intensivo, } \\
\text { multivariado, } \\
\text { transeccional. }\end{array}$ & $\begin{array}{l}\text { Expresiones } \\
\text { humorísticas y } \\
\text { risueñas. }\end{array}$ & $\begin{array}{l}19 \text { niños con autismo de entre } 36 \text { y } \\
65 \text { meses de edad y } 16 \text { niños con } \\
\text { síndrome de Down de entre } 18 \text { y } \\
65 \text { meses de edad. }\end{array}$ & $\begin{array}{l}\text { La mayoría de los sujetos con autismo } \\
\text { no encuentra graciosos los actos } \\
\text { sociales inapropiados ni las caras } \\
\text { graciosas. Un } 88 \% \text { de los sujetos con } \\
\text { autismo suele reírse sin razón } \\
\text { aparente. }\end{array}$ \\
\hline $\begin{array}{l}\text { Reddy, } \\
\text { Williams, } \\
\text { Costantini \& } \\
\text { Lan (2010) }\end{array}$ & $\begin{array}{l}\text { Descriptivo, } \\
\text { intensivo, } \\
\text { multivariado, } \\
\text { transversal. }\end{array}$ & $\begin{array}{l}\text { Conducta frente al } \\
\text { espejo. }\end{array}$ & $\begin{array}{l}12 \text { niños con autismo de } 47 \text { meses } \\
\text { de edad media, } 13 \text { con síndrome } \\
\text { de Down de } 42 \text { meses de edad } \\
\text { media y } 13 \text { con desarrollo típico de } \\
18 \text { meses de edad media. }\end{array}$ & $\begin{array}{l}\text { A diferencia de lo ocurrido con los } \\
\text { grupos control, la mayoría de los } \\
\text { sujetos con autismo no realiza } \\
\text { acciones sociales frente al espejo (e.g., } \\
\text { saludarse) y exhibe menor grado de } \\
\text { afecto positivo dirigido hacia su propio } \\
\text { rostro. }\end{array}$ \\
\hline
\end{tabular}

De modo similar, en otros tres estudios se concluye que los sujetos con autismo son menos propensos a efectuar gestos verbales y no verbales espontáneos de saludo y a establecer contacto visual, incluso de forma motivada (Hobson \& Lee, 1998), muestran menos preocupación ante una persona angustiada (Hobson et al., 2009) y tienen dificultades para involucrarse con ellos mismos al mirarse en el espejo (Reddy et al., 2010). 
Por último, en otro estudio García-Pérez et al. (2007) estudiaron la comunicación no verbal en adolescentes con y sin autismo a partir de entrevistas semiestructuradas de 60 minutos de duración, de las cuales se extrajeron los 3 minutos más significativos. Los resultados revelan ciertas características diferenciales del involucramiento entrevistador/sujeto con autismo que se agrupan según un conjunto de medidas comportamentales (e.g., dirección y duración de la mirada, movimientos de cabeza, cantidad y tipo de sonrisas) e intersubjetivas (e.g., involucramiento afectivo y fluidez de la entrevista). Así, por ejemplo, mientras el entrevistador habla, los niños con autismo lo miran menos que los niños con desarrollo típico. Además, mientras que el grado de involucramiento afectivo de los niños sin diagnóstico de autismo es mayoritariamente moderado o alto, el grado de involucramiento afectivo de los niños con autismo es nulo, mínimo o moderado. Por último, el estudio revela que la entrevista con los sujetos sin diagnóstico de autismo se desarrolla de un modo razonablemente fluido o muy fluido. En tanto, la mayor parte de las entrevistas con los sujetos con autismo exhibe menores grados de fluidez, que abarcan desde mínimamente fluida hasta un poco fluida. Los sujetos con autismo parecerían presentar, entonces, severas dificultades a la hora de producir y percibir señales sociales relevantes para lograr un involucramiento social fluido y afectivo.

En resumen, los resultados muestran que los sujetos con autismo presentan problemas en el reconocimiento emocional (Hobson et al., 1988) y severas dificultades a la hora de producir y percibir signos sociales relevantes para lograr un involucramiento social fluido y afectivo (Hobson \& Lee, 1998; Hobson et al. 2009; Reddy et al., 2002; Reddy et al., 2010) que podrían explicarse como producto de una diferencial comprensión intencional, enactiva y no inferencial.

\section{Metodología de la Investigación desde la P2P}

Aceptar los principios teóricos de la P2P implica, como hemos mencionado en secciones anteriores, una incompatibilidad profunda respecto de los principios metodológicos tradicionalmente empleados por la psicología. Ello es así porque la metodología psicológica tradicional y la metodología que se derivaría de la $\mathrm{P} 2 \mathrm{P}$ suponen visiones del mundo contradictorias. Tradicionalmente ha primado en psicología una metodología individualista, anclada en una visión realista del mundo, y asociada al método experimental como recurso privilegiado de acceso al conocimiento (Reddy \& Morris, 2004; Valsiner, Molenaar, Lyra \& Chaudhary, 2009). La P2P contradice esta perspectiva, en virtud de su talante intersubjetivista, su compromiso con una visión del mundo realista crítico y la valoración de los estudios empíricos ecológicos como forma privilegiada de acceso al saber. Recientemente, varios autores (Alessandroni et al., 2016; De Jaegher et al., 2010; Reddy, 2001a, 2010, 2011; Reddy \& Trevarthen, 2004) plantearon la necesidad de repensar los principios metodológicos clásicos, cuestionando a los lineamientos metodológicos asociados a las perspectivas de primera y de tercera persona. Las críticas apuntan a que en dichas perspectivas:

1. Las exploraciones empíricas se cimientan en el principio de ajenidad del investigador respecto de los sujetos investigados.

2. Entre los abordajes empíricos predomina el enfoque experimental, que crea situaciones extremadamente controladas que carecen de validez externa.

3. Se emplean exclusivamente variables intrapersonales.

Sin embargo, parecería no estar del todo claro de qué modo estudiar la atribución mentalista si para ello debemos centrarnos en procesos interactivos de involucramiento intersubjetivo y no en las respuestas que brindan los sujetos ante estímulos experimentales en contextos artificiales y controlados. Dicho de otro modo, no queda claro qué modificaciones en el sistema categorial de la metodología de la investigación entraña la adopción de esta perspectiva ni qué características debería poseer una genuina metodología de segunda persona (en adelante M2P). En una propuesta anterior (Alessandroni et al., 2016) hemos distinguido entre una M2P en sentido amplio y una en sentido estricto. Debido al posible impacto de la propuesta, en los párrafos sucesivos examinamos estas dos formas de M2P.

Comprometerse con la P2P implica, mínimamente, acordar en que los sujetos no se encuentran aislados unos de otros, sino involucrados entre sí. Investigar sujetos es, así, sinónimo de estudiar sujetos en interrelación en ámbitos ecológicos. La M2P en sentido amplio plantea que este objetivo puede lograrse considerando variables interpersonales o de segunda persona en el proceso de investigación. Ellas se diferencian de las variables intrapersonales en que no son propiedades de individuos (e.g., edad), sino de unidades de análisis quiasmáticas (e.g., ritmo de interacción). La unidad de análisis a la que refieren las variables de segunda persona desborda los límites de la subjetividad individual y refiere, en cambio, a una unidad dual formada a partir del entrelazamiento interactivo de dos sujetos. Por ejemplo, la fluidez de la interacción no es una 
propiedad de ninguno de los individuos que interactúan ni tampoco de la yuxtaposición de ambos sujetos, sino de la relación de involucramiento que se establece entre ellos. Además, estas variables poseen valores relacionales, que no consisten en la adición de la participación de cada uno de los sujetos que interactúan, es decir, que no son una función de los grados de responsabilidad parciales que cada sujeto aporta al proceso o propiedad que se desea medir. Por último, estas variables requieren indicadores de segunda persona, es decir, procedimientos que permitan al investigador penetrar en una dimensión de la variable intersubjetiva en la que está interesado sin transformar su esencia procesual y quiasmática.

Sería posible pensar, sin embargo, que la consideración de variables de segunda persona no constituye un giro suficientemente radical. La M2P en sentido estricto afirma esto y plantea que no alcanza con observar los procesos de involucramiento intersubjetivo, sino que es necesario ser parte de ellos. La propuesta responde a que, desde el punto de vista de la P2P, el compromiso emocional con otros sujetos nos brinda acceso a información que, de otro modo, no estaría disponible para nosotros (Reddy, 2010). No obtenemos la misma información al interactuar con alguien que al observarlo objetivamente. Según Reddy (2010, 2011), este principio teórico debería extenderse a los enfoques metodológicos para la investigación psicológica. En sintonía con este planteo, De Jaegher et al. (2010) consideran que "el estudio del compromiso puede exigir el desarrollo de metodologías de segunda persona, en las que el experimentador intervenga directamente como participante en la interacción" (p. 442).

Como hemos señalado anteriormente, Bordoni et al. (2016) apelan a esta metodología a la hora de recolectar sus datos (véase la subsección Estudios Empíricos en Desarrollo Típico). A pesar del impacto que podrían tener para la epistemología de la metodología de la investigación en psicología, son muy pocos los emprendimientos empíricos llevados adelante desde esta perspectiva, cuestión que señala la importancia de este tópico al interior de la agenda psicológica.

\section{Críticas a la P2P}

En las secciones anteriores hemos visto una serie de innovaciones teóricas y metodológicas interesantes, derivadas de la adopción de la P2P. No obstante, es posible circunscribir un conjunto de críticas y cuestionamientos a esta perspectiva. Algunos son internos, es decir, solicitan mayores niveles de precisión sobre puntos oscuros de la P2P, sin renunciar a la cosmovisión asociada a ella, a sus principios ontológicos, metodológicos y axiológicos ni a sus fundamentos epistemológicos. Otros son externos, es decir, introducen un mayor nivel de disonancia, pues su resolución (y a veces su formulación misma) desafía los principios epistemológicos y premisas teóricas que rigen a la $\mathrm{P} 2 \mathrm{P}$.

\section{Críticas y cuestionamientos internos.}

- Falta de una integración coherente con otras formas de AEM. En la literatura suele afirmarse que el tipo de AEM propuesto por la P2P es ontogenéticamente anterior (y podría convivir con) a aquellos planteados por las perspectivas de primera y de tercera persona (Gomila, 2001, 2002, 2008; Scotto, 2002). Esto implica, por un lado, que la $\mathrm{P} 2 \mathrm{P}$ no sería un corpus teórico autosuficiente y, en virtud de ello, que hace falta una integración ontogenéticamente coherente de las tres perspectivas sobre la AEM (Alessandroni et al., 2017; Riquelme, Henríquez y Álvarez, 2003).

- La P2P no estudia lo mismo que las otras perspectivas de AEM. Se ha señalado que la P2P, a pesar de pretenderse superadora de las perspectivas ortodoxas, provee explicaciones sobre un explanandum diferente al que estas últimas pretenden abordar (Dullstein, 2012). Al deflacionar excesivamente el concepto de AEM, la P2P aborda elementos (por ejemplo, gestos y movimientos) que hacen que este concepto pierda especificidad y, por ende, deje de tener valor heurístico.

- Falta de evidencia empírica a favor de la P2P. Hasta la fecha, los emprendimientos empíricos enmarcados en la P2P son escasos y metodológicamente diversos. La conducción de un mayor número de estudios permitiría precisar la propuesta teórica de la $\mathrm{P} 2 \mathrm{P}$, delimitar los lineamientos metodológicos asociados a ella y revisar sus compromisos epistemológicos.

- Las investigaciones empíricas deben encararse desde una M2P. Es necesario que exista correspondencia entre la propuesta teórica de la P2P y la metodología utilizada en los estudios realizados desde esta perspectiva (Alessandroni et al., 2016). Si es verdad que en una interacción existe información valiosa a la que solo puede accederse formando parte de ella (i.e., ingresando al campo de lo personal del otro) (Reddy, 2010), entonces: (a) la recolección de datos deberá contemplar, como indica la M2P en sentido estricto, la interacción del investigador con los sujetos de la investigación y (b) el análisis de los datos no 
puede pensarse como un segundo momento, desvinculado de la interacción, en el que el investigador objetiva aquello observado. Deben pensarse alternativas, que puedan abarcar, por ejemplo, la discusión acerca de la pertinencia de un análisis de datos simultáneo a la interacción y que incluya el testimonio de los sujetos interactuantes como término fundamental.

\section{Críticas y cuestionamientos externos.}

- En el mundo no existen relaciones diádicas puras. En la P2P la modalidad paradigmática de AEM son las interacciones diádicas cara a cara (Gomila, 2001). Sin embargo, este tipo de interacciones no existen en el mundo de modo puro. Las interacciones humanas habitualmente involucran a más de un sujeto y no siempre siguen la forma de intercambios cara a cara. Además, ellas siempre tienen lugar en un entorno materialmente significante. Dicho de otro modo, las interacciones humanas son siempre triádicas (involucran objetos), aun cuando en el comienzo la responsabilidad de la triadicidad sea del adulto (Rodríguez, Benassi, Estrada y Alessandroni, 2017).

- La cognición social desde la P2P es discontinua. Si la cognición social es equivalente al involucramiento en situaciones interactivas diádicas cara a cara, entonces nuestra cognición social temprana sería dependiente siempre de la interacción real en el mundo. De este modo, ella estaría limitada por los límites temporales y materiales que la interacción efectiva impone, de lo cual se sigue que nuestra comprensión de los otros sociales sería discontinua (solo se daría durante las interacciones diádicas) (Alessandroni, 2017, Enero).

- La P2P no explica los malos entendidos ni las divergencias interpretativas entre dos o más sujetos. Si aceptamos que los estados "mentales" pueden hallarse en las articulaciones corporales expresivas de los sujetos y que su percepción es directa, no se explica cómo son posibles los malos entendidos y las divergencias interpretativas entre dos o más sujetos para una misma situación interactiva. ¿Quién no ha "visto", casi con certeza, una emoción en el rostro de alguien cercano para luego percatarse de que dicha percepción era errada? El ejemplo indica que tal vez sea necesario interpelar el supuesto carácter directo de la percepción enactiva de los estados mentales. Dicho de otro modo, parecería ser que los criterios normativos de corrección de la acción no están en la acción misma: no existe nada en las expresiones emocionales-gestuales del otro que permita aseverar si las atribuciones mentalistas corporeizadas son o no correctas.

- La percepción no es directa. La P2P propone que la mente no es una dimensión abstracta u opaca del ser que contiene estados mentales inobservables, sino el modo en que un cuerpo viviente actúa en el mundo (Reddy, 2010). Las AEM se vuelven equivalentes, entonces, a la percepción directa e inmediata de las articulaciones corporales expresivas del otro. Este giro tiene, al menos, dos problemas. El primero de ellos es que podría interpretarse como una forma de reduccionismo, toda vez que involucraría un retorno a una forma de conductismo interesada por desentrañar las características de una interacción en términos de secuencias estímulo-respuesta perceptuales, empíricamente contrastables (Balmaceda, 2014). Pero también es posible negar que la percepción de las articulaciones corporales sea directa. De acuerdo con Alessandroni (2017, Enero), es razonable pensar que puede existir un modo de AEM no reflexivo ni teórico como el que propone la P2P, pero de allí no se sigue que la AEM sea un proceso natural, directo e inmediato. Basándose en el modelo semiótico social de Halliday (1978/2013), Alessandroni (2017, Enero) propone que la AEM es, en la temprana infancia, un proceso no teórico, pero sí semiótico, porque involucra instancias de interpretación que conforman progresivamente un potencial de significados que se apoya en el potencial de acción del niño. En otras palabras, el cuerpo nunca puede ser percibido directamente, porque su ontología excede la materialidad. El cuerpo es un terreno de significaciones sociales y, por ello, el significado que se construye en él es siempre dependiente de la significación de otro y de una semiótica social de orden superior. Esta crítica constituye una objeción a la totalidad, porque cuestiona un axioma de la P2P: la percepción de estados mentales es directa, básica y no está mediada por significaciones sociales. Por ende, no se trata de una crítica dirigida a perfeccionar la propuesta de la P2P, sino más bien a considerar enfoques alternativos (Alessandroni \& Rodríguez, 2018).

\section{Conclusiones}

En el campo específico de la AEM, la P2P resulta una alternativa novedosa a las perspectivas de primera y tercera persona, porque apela a una serie de recursos emocionales y prelingüísticos (por ejemplo, gestos o disposiciones corporales) para explicar la dinámica de la comprensión intencional temprana. Mientras que 
en las perspectivas ortodoxas el acceso a las otras mentes depende de una capacidad representacionalinferencial que no emerge hasta por lo menos los 2 años de edad, la AEM propuesta por la P2P no requiere de capacidades representacionales ni de inferencia alguna, siendo así más primigenia ontogenéticamente y más parsimoniosa en el terreno epistemológico. Así, la P2P tiene la ventaja de trazar una imagen del desarrollo de la AEM mucho más armónica que la propuesta por otras teorías, ya que no implica discontinuidades agudas en el desarrollo de la comprensión de otras mentes (i.e., las mentes son accesibles desde la temprana infancia). Además, la P2P da un lugar preponderante al cuerpo en el campo de la cognición social. Ello permite ampliar y complejizar el campo de la AEM, rompiendo con una larga tradición reduccionista subsidiaria del dualismo cartesiano.

A través de la revisión de estudios empíricos sobre desarrollo típico y cognición social, hemos mostrado que en contextos de interacción intersubjetiva tiene lugar una serie de conductas que demostrarían la existencia de una comprensión recíproca entre el niño y el adulto de tipo intencional, enactiva y no inferencial. Asimismo, la revisión de estudios en psicopatología del desarrollo pone de manifiesto que los sujetos con autismo exhiben un conjunto de comportamientos diferenciales que podrían ser explicados apelando a diferencias estructurales y dinámicas en el tipo de involucramiento temprano en que estos sujetos pueden participar.

No hemos querido dejar de lado la revisión de aquello que poco a poco comienza a ser denominado como M2P. Hemos explicado, en primer lugar, por qué esta perspectiva metodológica implica un rechazo de los lineamientos ortodoxos para la investigación psicológica. En segundo lugar, hemos presentado las principales características de esta novel propuesta metodológica, diferenciando entre M2P en sentido amplio y en sentido estricto.

Por último, hemos enumerado un conjunto de críticas y cuestionamientos internos y externos que, lejos de intentar desarticular la $\mathrm{P} 2 \mathrm{P}$, podrían propiciar su enriquecimiento mediante la apertura a otros desarrollos teóricos y epistemológicos. Creemos que, si la P2P lograra tomar en consideración los señalamientos críticos, podría erigirse como un corpus teórico robusto para explicar la cognición social y su desarrollo ontogenético.

\section{Referencias}

Alessandroni, N. (2017, Enero). 'Hey, what's goin' on?': la atribución de estados mentales es un proceso semiótico-pragmático. Conferencia presentada en el Seminario del Equipo Desarrollo Temprano y Educación, Madrid, España.

Alessandroni, N. \& Rodríguez, C. (2018). On perception as the basis for object concepts: A critical analysis. Society for the Improvement of Psychological Science, PsyArXiv. https://doi.org/10.31234/osf.io/ce9yf

Alessandroni, N., Vietri, M. \& Krasutzky, I. (2017). Atribución de estados mentales y autismo: desarrollos teóricos y controversias actuales. En M. C. Piro (Ed.), El autismo: perspectivas teórico-clínicas y desafíos contemporáneos (pp. 103-150). La Plata, Argentina: Editorial de la Universidad Nacional de La Plata.

Alessandroni, N., Vietri, M. \& Piro, M. (2016). Elementos para una metodología de segunda persona para la investigación en desarrollo y cognición social. Temas en Psicología, 3, 31-47. Extraído de http://www.psico.unlp.edu.ar/uploads/docs/anuario_temas_en_psicologia_volumen_3.pdf

Ambrosini, E., Reddy, V., de Looper, A., Costantini, M., Lopez, B. \& Sinigaglia, C. (2013). Looking ahead: Anticipatory gaze and motor ability in infancy. PLoS One, 8(7), artículo e67916. https://.doi.org/10.1371/journal.pone.0067916

American Psychiatric Association (2013). Diagnostic and statistical manual of mental disorders: DSM 5 (5a ed.). Washington, DC: Autor.

Balmaceda, T. (2014). Psicología del sentido común: pasado, presente y futuros. Buenos Aires, Argentina: Título.

Baron-Cohen, S. (1985). Mindblindness: An essay on autism and theory of mind. Cambridge, MA: MIT Press.

Benjamin, J. (1990). An outline of intersubjectivity: The development of recognition. Psychoanalytic Psychology, 7(Suppl), 33-46. https://doi.org/10.1037/h0085258

Bordoni, M., Español, S. \& De Grande, P. (2016). La incidencia del entonamiento afectivo y la imitación en el involucramiento visualsocial temprano. Avances en Psicología Latinoamericana, 34, 487-503. https://doi.org/10.12804/apl34.3.2016.04

Buhrmann, T. \& Di Paolo, E. (2017). The sense of agency - a phenomenological consequence of enacting sensorimotor schemes. Phenomenology and the Cognitive Sciences, 16, 207-236. https://doi.org/10.1007/s11097-015-9446-7

Burdman, F. (2015). El post-cognitivismo en cuestión: extensión, corporización y enactivismo. Principia, 19, 475-495. https://doi.org/10.5007/1808-1711.2015v19n3p475

Cohn, J. F. \& Tronick, E. Z. (1987). Mother-infant face-to-face interaction: The sequence of dyadic states at 3, 6, and 9 months. Developmental Psychology, 23, 68-77. https://doi.org/10.1037/0012-1649.23.1.68

Costall, C., Leudar, I. \& Reddy, V. (2006). Failing to see the irony in 'mind-reading'. Theory \& Psychology, 16, 163-167. https://doi.org/10.1177/0959354306062533

Dawson, G., Hill, D., Spencer, A., Galpert, L. \& Watson, L. (1990). Affective exchanges between young autistic children and their mothers. Journal of Abnormal Child Psychology, 18, 335-345. https://doi.org/10.1007/BF00916569

De Jaegher, H. \& Di Paolo, E. (2007). Participatory sense-making: An enactive approach to social cognition. Phenomenology and the Cognitive Sciences, 6, 485-507. https://doi.org/10.1007/s11097-007-9076-9

De Jaegher, H., Di Paolo, E. \& Gallagher, S. (2010). Can social interaction constitute social cognition? Trends in Cognitive Sciences, 14, 441-447. https://doi.org/10.1016/j.tics.2010.06.009

Di Paolo, E. A. \& De Jaegher, H. (2015). Toward an embodied science of intersubjectivity: Widening the scope of social understanding research. Frontiers in Psychology, 6, 234. https://doi.org/10.3389/fpsyg.2015.00234 
Dominey, P. F., Prescott, T. J., Bohg, J., Engel, A. K., Gallagher, S., Heed, T. ... Schwartz, A. (2016). Implications of action-oriented paradigms shifts in cognitive science. En A. K. Engel, K. J. Friston \& D. Kragic (Eds.), The pragmatic turn: Toward action-oriented views in cognitive science (pp. 333-356). Cambridge, MA: MIT Press.

Dullstein, M. (2012). The second person in the theory of mind debate. Review of Philosophy and Psychology, 3, 231-248. https://doi.org/10.1007/s13164-012-0095-2

Español, S. (2007). Experiencia estética y desarrollo humano. Las artes temporales en la génesis de procesos psicológicos complejos. Psykhe, 16(1), 123-133. https://doi.org/10.4067/S0718-222820070001000252

Fantasia, V., De Jaegher, H. \& Fasulo, A. (2014). We can work it out: An enactive look at cooperation. Frontiers in Psychology, 5, 874. https://doi.org/10.3389/fpsyg.2014.00874

Fantasia, V., Fasulo, A., Costall, A. \& López, B. (2014). Changing the game: Exploring infants' participation in early play routines. Frontiers in Psychology, 5, 522. https://doi.org/10.3389/fpsyg.2014.00522

Fantasia, V., Markova, G., Fasulo, A., Costall, A. \& Reddy, V. (2015). Not just being lifted: Infants are sensitive to delay during a pickup routine. Frontiers in Psychology, 6, 2065. https://doi.org/10.3389/fpsyg.2015.02065

Ferrer de Luna, J. (2014). El papel de la segunda persona en la constitución del autoconocimiento. Daimon: Revista Internacional de Filosofía, 62, 71-85. https://doi.org/10.6018/daimon/164831

Gallagher, S. (2001). The practice of mind: Theory, simulation or interaction? En E. Thompson (Ed.), Between ourselves: Second person issues in the study of consciousness (pp. 83-108). Exeter, Reino Unido: Imprint Academic.

Gallagher, S. (2004). Understanding interpersonal problems in autism: Interaction theory as an alternative to theory of mind. Philosophy, Psychiatry, \& Psychology, 11, 199-217. https://doi.org/10.1353/ppp.2004.0063

Gallagher, S. (2008). Direct perception in the intersubjective context. Consciousness and Cognition, 17, 535-543. https://doi.org/10.1016/j.concog.2008.03.003

Gallagher, S. (2016). Intercorporeity: Enaction, simulation, and the science of social cognition. En J. Reynolds \& R. Sebold (Eds.), Phenomenology and science: Confrontations and convergences (pp. 161-179). New York, NY: Palgrave Macmillan.

Gallese, V. (2014). Bodily selves in relation: Embodied simulation as second-person perspective on intersubjectivity. Philosophical Transactions of the Royal Society B: Biological Sciences, 369(1644), artículo 20130177. https://doi.org/10.1098/rstb.2013.0177

García-Pérez, R. M., Lee, A. \& Hobson, R. P. (2007). On intersubjective engagement in autism: A controlled study of nonverbal aspects of conversation. Journal of Autism and Developmental Disorders, 37, 1310-1322. https://doi.org/10.1007/s10803-006-0276-x

Goldman, A. I. (1989). Interpretation psychologized. Mind \& Language, 4, 161-185. https://doi.org/10.1111/j.1468-0017.1989.tb00249.x

Goldman, A. \& Mason, K. (2007). Simulation. En P. Thagard (Ed.), Philosophy of psychology and cognitive science (pp. 267-293). Amsterdam, Países Bajos: Elsevier.

Gomila, A. (2001). La perspectiva de segunda persona: mecanismos mentales de la intersubjetividad. Contrastes: Revista Internacional de Filosofía, 6(Suplemento 6), 65-86. https://doi.org/10.24310/Contrastescontrastes.v0i0.1448

Gomila, A. (2002). La perspectiva de segunda persona de la atribución mental. Azafea: Revista de Filosofía, 4, 123-138. http://revistas.usal.es/index.php/0213-3563/article/view/3719/3736

Gomila, A. (2008). La relevancia moral de la perspectiva de segunda persona. En D. Pérez \& L. Fernández Moreno (Comps.), Cuestiones filosóficas. Ensayos en honor de Eduardo Rabossi (pp. 155-173). Buenos Aires, Argentina: Catálogos.

Gopnik, A. \& Wellman, H. M. (1994/2002). La teoría de la teoría (Título original: The theory theory). En L. A. Hirschfeld \& S. A. Gelman (Comps.), Cartografía de la mente: La especificidad de dominio en la cognición y en la cultura (A. Ruiz, Trad.; Título original: Mapping the mind: Domain specificity in cognition and culture; pp. 13-63). Barcelona, España: Gedisa.

Gordon, R. M. (1986). Folk psychology as simulation. Mind \& Language, 1, 158-171. https://doi.org/10.1111/j.1468-0017.1986.tb00324.x

Halliday, M. A. K. (1978/2013). El lenguaje como semiótica social: la interpretación social del lenguaje y del significado ( $1^{\mathrm{a}}$ ed., $4^{\mathrm{a}}$ reimp.; J. F. Santana, Trad.; Título original: Language as social semiotic: The social interpretation of language and meaning). México DF, México: Fondo de Cultura Económica.

Heal, J. (1986). Replication and functionalism. En J. Butterfield (Ed.), Language, mind and logic (pp. 135-150). New York, NY: Cambridge University Press.

Hobson, J. A., Harris, R., García-Pérez, R. \& Hobson, R. P. (2009). Anticipatory concern: A study in autism. Developmental Science, 12, 249-263. https://doi.org/10.1111/j.1467-7687.2008.00762.x

Hobson, P. (2002). The cradle of thought: Exploring the origins of thinking. London, Reino Unido: Macmillan.

Hobson, R. P. (1990). On acquiring knowledge about people and the capacity to pretend: Response to Leslie (1987). Psychological Review, 97, 114-121. https://doi.org/10.1037/0033-295X.97.1.114

Hobson, R. P. \& Lee, A. (1998). Hello and goodbye: A study of social engagement in autism. Journal of Autism and Developmental Disorders, 28, 117-127. https://doi.org/10.1023/A:1026088531558

Hobson, R. P. \& Lee, A. (1999). Imitation and identification in autism. Journal of Child Psychology and Psychiatry, 40, 649-659. https://doi.org/10.1111/1469-7610.00481

Hobson, R. P., Ouston, J. \& Lee, A. (1988). What's in a face? The case of autism. British Journal of Psychology, 79, 441-453. https://doi.org/10.1111/j.2044-8295.1988.tb02745.x

Hubley, P. \& Trevarthen, C. (1979). Sharing a task in infancy. New Directions for Child and Adolescent Development, 1979(4), 57-80. https://doi.org/10.1002/cd.23219790406

Hutto, D. D. (2008). Folk psychological narratives: The sociocultural basis of understanding reasons. Cambridge, MA: MIT Press.

Karmiloff-Smith, A. (1994). Beyond modularity: A developmental perspective on cognitive science. Cambridge, MA: MIT Press.

Kaye, K. \& Fogel, A. (1980). The temporal structure of face-to-face communication between mothers and infants. Developmental Psychology, 16, 454-464. https://doi.org/10.1037/0012-1649.16.5.454

Klin, A., Jones, W., Schultz, R. \& Volkmar, F. (2003). The enactive mind, or from actions to cognition: Lessons from autism. Philosophical Transactions of the Royal Society B: Biological Sciences, 358(1430), artículo 20021202. https://doi.org/10.1098/rstb.2002.1202

Leslie, A. M. (1987). Pretense and representation: The origins of "theory of mind". Psychological Review, 94, 412-426. https://doi.org/10.1037/0033-295X.94.4.412

Leslie, A. M. (2000). How to acquire a representational theory of mind. En D. Sperber (Ed.),Metarepresentations: A multidisciplinary perspective (pp. 197-223). New York, NY: Oxford University Press.

Leudar, I. \& Costall, A. (2004). On the persistence of the 'problem of other minds' in psychology: Chomsky, Grice and theory of mind. Theory \& Psychology, 14, 601-621. https://doi.org/10.1177/0959354304046175 
Mahler, M. S., Pine, F. \& Bergman, A. (1975/1977). El nacimiento psicológico del infante humano: simbiosis e individuación (E. J. Prieto, Trad.; Título original: The psychological birth of the human infant: Symbiosis and individuation). Buenos Aires, Argentina: Marymar.

Melot, A. M. \& Nadel, J. (1998/2003). Teoría de la mente (Título original: Théorie de l'esprit). En O. Houdé, D. Kayser, O. Koenig, J. Proust \& F. Rastier (Eds.), Diccionario de ciencias cognitivas: neurociencia, psicología, inteligencia artificial, lingüistica y filosofía (C. R. Molinari-Marotto, Trad.; Vocabulaire de sciences cognitives: Neuroscience, psychologie, intelligence artificielle, linguistique et philosophie; pp. 434-436). Buenos Aires, Argentina: Amorrortu.

Mojzisch, A., Schilbach, L., Helmert, J. R., Pannasch, S., Velichkovsky, B. M. \& Vogeley, K. (2006). The effects of self-involvement on attention, arousal, and facial expression during social interaction with virtual others: A psychophysiological study. Social Neuroscience, 1, 184-195. https://doi.org/10.1080/17470910600985621

Newton, P., Reddy, V. \& Bull, R. (2000). Children's everyday deception and performance on false-belief tasks. British Journal of Developmental Psychology, 18, 297-317. https://doi.org/10.1348/026151000165706

Pedace, K. (2012). La normatividad de lo mental y el rol de la segunda persona. Tras las huellas de Donald Davidson. Areté: Revista de Filosofía, 24, 109-152. Extraído de http://revistas.pucp.edu.pe/index.php/arete/article/view/2734

Pérez, D. (2013). Sentir, desear, creer. Una aproximación filosófica a los conceptos psicológicos. Buenos Aires, Argentina: Prometeo.

Pérez, D. \& Español, S. (2014). Intersubjetividad y atribución psicológica. En P. Quintanilla, C. Mantilla \& P. Cépeda (Eds.), Cognición social y lenguaje: La intersubjetividad en la evolución de la especie y en el desarrollo del niño (pp. 371-392). Lima, Perú: Fondo Editorial de la Pontificia Universidad Católica del Perú.

Pérez, D. \& Lawler, D. (Comps.) (2017). La segunda persona y las emociones. Buenos Aires, Argentina: Sociedad Argentina de Análisis Filosófico.

Piccinini, G. (2007). Computational explanation and mechanistic explanation of mind. En M. Marraffa, M. De Caro \& F. Ferretti (Eds.), Cartographies of the mind: Philosophy and psychology in intersection (pp. 23-36). Dordrecht, Países Bajos: Springer. https://doi.org/10.1007/1-4020-5444-0_2

Premack, D. \& Woodruff, G. (1978). Does the chimpanzee have a theory of mind? Behavioral and Brain Sciences, 1, 515-526. https://doi.org/10.1017/S0140525X00076512Ranly, E. W. (1966). Man's knowledge of man. En E. W. Ranly, Scheler's phenomenology of community (pp. 38-61). Den Haag, Países Bajos: Martinus Nijhoff. https://doi.org/10.1007/978-94-015-0844-5

Reddy, V. (2000). Coyness in early infancy. Developmental Science, 3(2), 186-192. https://doi.org/10.1111/1467-7687.00112

Reddy, V. (2001a). Mind knowledge in the first year: Understanding attention and intention. En G. Bremner \& A. Fogel (Eds.), Blackwell handbook of infant development (pp. 241-264). Malden, MA: Blackwell.

Reddy, V. (2001b). Infant clowns: The interpersonal creation of humour in infancy. Enfance, 53, 247-253. https://doi.org/10.3917/enf.533.0247

Reddy, V. (2003). On being the object of attention: Implications for self-other consciousness. Trends in Cognitive Sciences, 7, 397-402. https://doi.org/10.1016/S1364-6613(03)00191-8

Reddy, V. (2010). How infants know minds. Cambridge, MA: Harvard University Press.

Reddy, V. (2011). A gaze at grips with me. En A. Seemann (Ed.), Joint attention: New developments in psychology, philosophy of mind, and social neuroscience (pp. 137-157). Cambridge, MA: MIT Press.

Reddy, V., Liebal, K., Hicks, K., Jonnalagadda, S. \& Chintalapuri, B. (2013). The emergent practice of infant compliance: An exploration in two cultures. Developmental Psychology, 49, 1754-1762. https://doi.org/10.1037/a0030979

Reddy, V., Markova, G. \& Wallot, S. (2013). Anticipatory adjustments to being picked up in infancy. PLoS One, 8(6), artículo e65289. https://doi.org/10.1371/journal.pone.0065289

Reddy, V. \& Morris, P. (2004). Participants don't need theories: Knowing minds in engagement. Theory \& Psychology, 14, 647-665. https://doi.org/10.1177/0959354304046177

Reddy, V. \& Trevarthen, C. (2004). What we learn about babies from engaging with their emotions. Zero to Three, 24(3), 9-15. Extraído de https://researchportal.port.ac.uk/portal/en/searchall.html?searchall=What+we+learn+about+babies+from+engaging+their+emotions

Reddy, V. \& Uithol, S. (2016). Engagement: Looking beyond the mirror to understand action understanding. British Journal of Developmental Psychology, 34, 101-114. https://doi.org/10.1111/bjdp.12106

Reddy, V., Williams, E., Costantini, C. \& Lan, B. (2010). Engaging with the self: Mirror behaviour in autism, Down syndrome and typical development. Autism, 14, 531-546. https://doi.org/10.1177/1362361310370397

Reddy, V., Williams, E. \& Vaughan, A. (2002). Sharing humour and laughter in autism and Down's syndrome. British Journal of Psychology, 93, 219-242. https://doi.org/10.1348/000712602162553

Riquelme, E. H., Henríquez, C. \& Álvarez, B. (2003). Relación entre estilos de apego y teoría de la mente. Psykhe, 12(1), 73-81. Extraído de http://www.psykhe.cl/index.php/psykhe/article/view/342

Rodríguez, C., Benassi, J., Estrada, L. \& Alessandroni, N. (2017). Early social interactions with people and objects. En A. Slater \& J. Bremner (Eds.), An introduction to developmental psychology ( $3^{a}$ ed., pp. 213-258). Hoboken, NJ: John Wiley \& Sons.

Schilbach, L., Eickhoff, S. B., Cieslik, E. C., Kuzmanovic, B. \& Vogeley, K. (2012). Shall we do this together? Social gaze influences action control in a comparison group, but not in individuals with high-functioning autism. Autism, 16, 151-162. https://doi.org/10.1177/1362361311409258

Schilbach, L., Eickhoff, S. B., Cieslik, E., Shah, N. J., Fink, G. R. \& Vogeley, K. (2011). Eyes on me: An fMRI study of the effects of social gaze on action control. Social Cognitive and Affective Neuroscience, 6, 393-403. https://doi.org/10.1093/scan/nsq067

Schilbach, L., Timmermans, B., Reddy, V., Costall, A., Bente, G., Schlicht, T. \& Vogeley, K. (2013). Toward a second-person neuroscience. Behavioral and Brain Sciences, 36, 393-414. https://doi.org/10.1017/S0140525X12000660

Schilbach, L., Wilms, M., Eickhoff, S. B., Romanzetti, S., Tepest, R., Bente, G. ... Vogeley, K. (2010). Minds made for sharing: Initiating joint attention recruits reward-related neurocircuitry. Journal of Cognitive Neuroscience, 22, 2702-2715. https://doi.org/10.1162/jocn.2009.21401

Schilbach, L., Wohlschlaeger, A. M., Kraemer, N. C., Newen, A., Shah, N. J., Fink, G. R. \& Vogeley, K. (2006). Being with virtual others: Neural correlates of social interaction. Neuropsychologia, 44, 718-730. https://doi.org/10.1016/j.neuropsychologia.2005.07.017

Schutz, A. (1962). Scheler's theory of intersubjectivity and the general thesis of the alter ego. En M. Natanson (Ed.), The problem of social reality (Collected Papers I, pp. 150-179). Den Haag, Países Bajos: Martinus Nijhoff. https://doi.org/10.1007/978-94-017-1077-0_6

Scotto, C. (2002). Interacción y atribución mental: la perspectiva de segunda persona. Análisis Filosófico, 22, $135-151$.

Shapiro, L. (2011). Embodied cognition. New York, NY: Routledge.

Sodian, B., Taylor, C., Harris, P. L. \& Perner, J. (1991). Early deception and the child's theory of mind: False trails and genuine markers. Child Development, 62, 468-483. https://doi.org/10.1111/j.1467-8624.1991.tb01545.x 
Somerville, J. A., Woodward, A. L. \& Needham, A. (2005). Action experience alters 3-month-old infants' perception of others' actions. Cognition, 96, B1-B11. https://doi.org/10.1016/j.cognition.2004.07.004

Spitz, R. A. (1965/1998). El primer año de vida del niño (12 ${ }^{\mathrm{a}}$ ed.; M. De la Escalera, Trad.; Título original: La premiére année de la vie de l'enfant). México DF, México: Fondo de Cultura Económica.

Stern, D. (1985/2005). El mundo interpersonal del infante: una perspectiva desde el psicoanálisis y la psicología evolutiva $\left(1^{\mathrm{a}}\right.$ ed., $4^{\mathrm{a}}$ reimpresión; J. Piatigorsky, Trad.; Título original: The interpersonal world of the infant: A view from psychoanalysis and developmental psychology). Buenos Aires, Argentina: Paidós.

Stouthamer-Loeber, M. (1986). Adults' perception of verbal misrepresentations of reality in four-year-olds. Manuscrito no publicado, University of Pittsburgh, PA, Estados Unidos.

Thelen, E. \& Smith, L. B. (1996). A dynamic systems approach to the development of cognition and action (1ª reimpresión). Cambridge, MA: MIT Press.

Trevarthen, C. (1979). Communication and cooperation in early infancy: A description of primary intersubjectivity. En M. Bullowa (Ed.), Before speech: The beginning of interpersonal communication (pp. 321-348). New York, NY: Cambridge University Press.

Trevarthen, C. (1984). Emotions in infancy: Regulators of contacts and relationships with persons. En K. R. Scherer y P. Ekman (Eds.), Approaches to emotion (pp. 129-162). Hillsdale, NJ: Lawrence Erlbaum.

Urrútia, G. \& Bonfill, X. (2010). Declaración PRISMA: una propuesta para mejorar la publicación de revisiones sistemáticas y metaanálisis. Medicina Clínica, 135, 507-511. https://doi.org/10.1016/j.medcli.2010.01.015

Valsiner, J., Molenaar, P. C. M., Lyra, M. C. D. P. \& Chaudhary, N. (Eds.) (2009). Dynamic process methodology in the social and developmental sciences. New York, NY: Springer. https://doi.org/10.1007/978-0-387-95922-1

Van der Maas, H. L. J. \& Molenaar, P. C. M. (1996). Catastrophe theory. En von Eye, A. \& Clogg, C. C. (Eds.), Categorical variables in developmental research: Methods of analysis (pp. 77-106). San Diego, CA: Academic Press. https://doi.org/10.1016/B978-0-12-724965-0.X5000-7

Wimmer, H. \& Perner, J. (1983). Beliefs about beliefs: Representation and constraining function of wrong beliefs in young children's understanding of deception. Cognition, 13, 103-128. https://doi.org/10.1016/0010-0277(83)90004-5

World Health Organization (2010). ICD-10: International statistical classification of diseases and related health problems (10a rev., $4^{\mathrm{a}}$ ed.). La Valeta, Malta: Autor.

Yeo, R. S. (2016). Renewing spiritual perception with Jonathan Edwards: Contemporary philosophy and the theological psychology of transforming grace. Abingdon, Reino Unido: Routledge.

Ynoub, R. (2015). Cuestión de método: aportes para una metodología crítica. México DF, México: Cengage Learning.

Zahavi, D. \& Parnas, J. (2003). Conceptual problems in infantile autism research: Why cognitive science needs phenomenology. Journal of Consciousness Studies, 10(9-10), 53-71. Extraído de https://www.ingentaconnect.com/content/imp/jcs/2003/00000010/f0020009/art00005

Zahn-Waxler, C. \& Radke-Yarrow, M. (1990). The origins of empathic concern. Motivation and Emotion, 14, 107-130. https://doi.org/10.1007/BF00991639

Fecha de recepción: Septiembre de 2017.

Fecha de aceptación: Diciembre de 2018. 